\title{
Prevalence of Chlamydia trachomatis infection in homosexual men
}

\author{
M Z C SULAIMAN,* J FOSTER, $\dagger$ AND S F PUGH $\ddagger$ \\ From the *Department of Genitourinary Medicine, General Hospital, $\ddagger$ Department of Microbiology and \\ Public Health Laboratory, and $†$ Medical School, University Hospital, Nottingham
}

SUMMARY Chlamydial cell culture isolation rates from 51 unselected homosexual men were 9.3\% from the urethra, $4.3 \%$ (pharynx), and $6.5 \%$ (rectum). All the positive cultures showed extremely few inclusions. Direct immunofluorescence with the Microtrak reagent (Syva) was an acceptable alternative technique for non-genital sites, whereas the infectious disease enzyme immunoassay (IDEIA) (Boots-Celltech) was unsuitable.

\section{Introduction}

Infections due to Chlamydia trachomatis are often sexually transmitted. Isolation of $C$ trachomatis from the rectum of unselected homosexual men varies from $4 \%$ to $8 \% .^{1-4} \mathrm{McMillan}$ et al isolated chlamydiae from the urethra of $10(7 \%)$ and from the pharynx of two (1\%) of 150 consecutive homosexual men who attended a sexually transmitted diseases clinic during two years. ${ }^{1}$ These studies used conventional tissue culture isolation of $C$ trachomatis. Newer methods of antigen detection by direct fluorescence and enzyme immunoassay have been used to improve diagnosis in men with urethritis and in women with cervicitis. In this study we compared the detection of chlamydiae from the urethra, pharynx, and rectum of homosexual men by three different methods: cell culture, amplified infectious disease enzyme immunoassay (IDEIA, BootsCelltech), and direct immunofluorescence.

\section{Patients and methods}

We obtained specimens from 51 unselected homosexual or bisexual men who attended the department of genitourinary medicine in Nottingham from September to December 1985 . We recorded the nature and extent of any urethral discharge and symptoms and signs relating to the pharynx and rectum. Urethral material was obtained by inserting a cotton tipped aluminium wire about $3 \mathrm{~cm}$ into the urethra and rotating it before removal. Pharyngeal material was taken by firmly

Address for reprints: Dr M Z C Sulaiman, Department of Genitourinary Medicine, Royal Hallamshire Hospital, Glossop Road, Sheffield S10 2JF

Accepted for publication 24 October 1986. scraping both sides of the posterior pharynx with a cotton tipped compressed paper stick (Minitip, Exogen). Rectal specimens were collected under direct vision through a lightly lubricated disposable proctoscope, by abrading the mucosa with a cotton tipped wooden swab.

Duplicate swabs were taken from each site. The swab for cell culture and IDEIA was placed in chlamydial transport medium, transported to the laboratory on ice, and stored at $4^{\circ} \mathrm{C}$ if it was processed the same day or at $-70^{\circ} \mathrm{C}$ if prolonged storage was necessary. The swab for direct immunofluorescence was rolled firmly in a $6 \mathrm{~mm}$ well on teflon coated slides. When the material was air dry it was fixed in acetone for at least five minutes and stored at $4^{\circ} \mathrm{C}$ until it was processed. The order in which the swabs were taken was reversed fortnightly.

\section{LABORATORY METHODS}

Specimens were cultured for chlamydiae as described previously. ${ }^{5}$ After innoculation the remainder of each specimen was stored at $-70^{\circ} \mathrm{C}$ for testing by IDEIA. Initially swabs from all three sites were examined by IDEIA, but this method was abandoned for rectal specimens because of a high false positive rate. Direct immunofluorescence was performed using the Microtrak reagent (Syva). The presence of epithelial cells indicated that an adequate specimen had been taken.

\section{Results}

Of the 51 patients in the study, 40 yielded specimens from all three sites. A further three urethral, seven pharyngeal, and seven rectal specimens were obtained from the remaining 11 patients. Eight pharyngeal and 
11 rectal specimens gave inadequate material for direct immunofluorescence, and one rectal specimen was too contaminated with faeces to use in cell culture.

The first 20 rectal swabs were examined by IDEIA and 11 gave positive results, which suggested a cross reaction with a component of faeces. To investigate this, extracts from 12 faecal samples submitted for viral culture $(10 \%$ weight/volume in phosphate buffered saline) were tested by IDEIA. All gave positive results, and this reactivity was not destroyed by boiling for one hour. We assumed that the false positive results obtained from rectal swabs reflected the amount of faecal material on the swabs, and therefore stopped using the IDEIA for rectal specimens.

The table compares results obtained by the three methods (excluding the IDEIA for rectal specimens) in the 11 patients who gave positive results from at least one anatomical site by at least one method. $C$ trachomatis was isolated by cell culture from the urethra of four $(9 \%)$ of $\mathbf{4 3}$ men, from the pharynx of two (4\%) of 47 men, and from the rectum of three (7\%) of 46 men. The IDEIA proved to be insensitive compared with culture because only two out of four culture positive urethral specimens were positive by IDEIA, and neither of the culture positive pharyngeal specimens were positive by this test. In addition, one out of three IDEIA positive urethral specimens and both IDEIA positive pharyngeal specimens gave negative results by the other two methods. Regarding direct immunofluorescence, two or more elementary bodies were seen in three of the four culture positive urethral specimens and in all the culture positive pharyngeal and rectal specimens. Elementary bodies were also seen in two culture negative pharyngeal and two culture negative rectal specimens.

Chlamydiae were isolated by cell culture from two
(50\%) of four men with symptomatic urethritis and from two (6\%) of 35 men without urethral symptoms; from one $(14 \%)$ of seven men with symptomatic pharyngitis and from one (3\%) of 40 men without pharyngeal symptoms; and from one $(25 \%)$ of four men with symptomatic proctitis and from two $(5 \%)$ of 42 men without rectal symptoms.

\section{Discussion}

Our chlamydial culture isolation rates from the urethras, pharynxes, and rectums of homosexual men were similar to those reported in previous studies. ${ }^{1-4}$ The low isolation rates at non-genital sites were possibly because of difficulties in obtaining good quality specimens from those sites. It was initially difficult to get adequate material from the rectal mucosa through the proctoscope, and this was only achieved when rigid wooden swabs were used instead of paper sticks.

All our chlamydiae positive cultures showed extremely few inclusions per slide, and few elementary bodies were seen on nearly all the positive direct immunofluorescence results. If at least 10 elementary bodies is taken as the cut off point, the specificity of direct immunofluorescence was $100 \%$, but several probable true positives would have been missed. Thomas et al consider that the morphology of an elementary body is so characteristic that a single elementary body in a urethral specimen is diagnostic. ${ }^{6}$ This may not be true of pharyngeal and rectal specimens, however. According to the manufacturers, a positive diagnosis of rectal specimens is based on seeing two or more elementary bodies, but they make no recommendations for pharyngeal specimens.

IDEIA was unsuitable for examining rectal swabs because a component of faeces cross reacted in the

TABLE Comparison of three methods of detecting Chlamydia trachomatis in 11 patients with positive results*

\begin{tabular}{|c|c|c|c|c|c|c|c|c|}
\hline \multirow[b]{2}{*}{ Case } & \multicolumn{3}{|c|}{ Results $*$ from urethra by: } & \multicolumn{3}{|c|}{ Results * from pharynx by: } & \multicolumn{2}{|c|}{ Results $*$ from rectum by: } \\
\hline & Culture & IDEIA & $D I F$ & Culture & IDEIA & $D I F$ & Culture & $D I F$ \\
\hline 1 & 4 & + & $>10$ & 0 & + & 0 & 3 & $>10$ \\
\hline 2 & 0 & - & 0 & 1 & - & 2 & 2 & $>10$ \\
\hline 3 & 2 & + & $>10$ & 0 & - & 0 & 0 & 0 \\
\hline 4 & 3 & - & 2 & 0 & - & 0 & 0 & 0 \\
\hline 5 & 2 & - & 0 & 1 & _- & 4 & 0 & 0 \\
\hline 6 & 0 & - & 0 & 0 & - & 0 & 2 & 4 \\
\hline 7 & 0 & - & 0 & 0 & 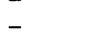 & 2 & 0 & $\begin{array}{l}4 \\
2\end{array}$ \\
\hline 8 & 0 & - & 0 & 0 & - & 8 & 0 & 0 \\
\hline 9 & 0 & - & 0 & 0 & + & 0 & 0 & 0 \\
\hline 10 & 0 & + & 0 & 0 & - & 0 & 0 & 0 \\
\hline 11 & 0 & - & 0 & 0 & - & 0 & 0 & 2 \\
\hline
\end{tabular}

* No of inclusions per slide on culture, positive (+) or negative (-) results on IDEIA (infectious disease enzyme immunoassay (Boots-Celltech), or No of elementary bodies per stide on direct immunofluorescence (DIF) using Microtrak reagent (Syva). 
test. The source of this cross reaction was not identified, though, as boiling for one hour did not reduce the reactivity, its source may be a polysaccharide antigen. Because confirmation of morphology is not possible by IDEIA, this method is probably unsuitable for specimens from the pharynx, where staphylococci may theoretically give a false positive result because of non-specific binding to protein A.

Several studies have shown that the chlamydial isolation rate from the urethras of homosexual men is less than that from heterosexual men, ${ }^{78}$ and that rectal infection associated with $C$ trachomatis is less common than urethritis caused by $C$ trachomatis. ${ }^{1-3}$ McMillan et al suggested that pharyngeal and rectal mucosa are less well suited than genital sites ${ }^{1}$ to colonisation by chlamydiae. ${ }^{1}$ Though the numbers of isolates obtained in our study were too small for us to draw any firm conclusions, we believe that direct immunofluorescence is an acceptable alternative technique to culture and that the IDEIA test is unsuitable for non-genital sites.

We thank Dr J B Bittiner for access to patients under his care and the staff of the Nottingham clinic and the PHLS laboratory for their co-operation. We also thank $\operatorname{Dr} G \mathrm{R}$
Kinghorn for his valuable comments and Miss Wendy Morris for her secretarial help.

\section{References}

1. McMillan A, Sommerville RG, McKie PMK. Chlamydial infection in homosexual men; frequency of isolation of Chlamydia trachomatis from the urethra, ano-rectum, and pharynx. British Journal of Venereal Diseases 1981;57:47-9.

2. Quinn TC, Goodell SE, Mkrtichian EC, et al. Chlamydia trachomatis proctitis. N Engl J Med 1981;305:195-200.

3. Munday PE,Dawson SG,Johnson AP, et al. A microbiological study of non-gonococcal proctitis in passive male homosexuals. Postgrad Med J 1981;57:705-11.

4. Munday PE, Carder JM, Taylor-Robinson D. Chlamydial proctitis. Genitourin Med 1985;61:376-8.

5. Pugh SF, Slack RCB, Caul EO, Paul ID, Appleton PN, Gatley S. Enzyme amplified immunoassay: a novel technique applied to direct detection of Chlamydia trachomatis in clinical specimens.J Clin Pathol 1985;38:1139-41.

6. Thomas BJ, Evans RT, Hawkins DA, Taylor-Robinson D. Sensitivity of detecting Chlamydia trachomatis elementary bodies in smears by use of a fluorescein labelled monoclonal antibody: comparison with conventional chlamydial isolation.J Clin Pathol 1984;37:812-6.

7. Goldmeir D, Darougar S. Isolation of Chlamydia trachomatis from throat and rectum of homosexual men. British Journal of Venereal Diseases 1977;53:184-5.

8. Bowie WR, Alexander ER, Holmes KK. Etiologies of postgonococcal urethritis in homosexual and heterosexual men: roles of Chlamydia trachomatis and Ureaplasma urealyticum. Sex Transm Dis 1978;5:151-4. 